\title{
ACTIVITY, STABILITY, AND STRUCTURE OF NATIVE AND MODIFIED BY WOODWARD REAGENT K MUSHROOM TYROSINASE
}

\author{
S. Emami, ${ }^{a}$ H. Piri, ${ }^{b}$ and N. Gheibi ${ }^{b^{*}}$
}

UDC 535.37:577.15

\begin{abstract}
Mushroom tyrosinase (MT) was considered a good model for studying the inhibition, activation, and mutation of tyrosinase as the key enzyme of melanogenesis. In the present study, the activity, structure, reduction, and stability of native and modified enzymes were investigated after the modification of MT carboxylic residues by the Woodward reagent $K$ (WRK). The relative activity of the sole enzyme was reduced from 100 to 77.9, 53.8, 39.4, and 26.4\% after its modification by $2.5,5,25$, and 50 ratios of [WRK]/[MT], respectively. The $T_{m}$ values were calculated from thermal denaturation curves at 61.2, 60.1, 58.3, 53.9, and $45.5^{\circ} \mathrm{C}$ for the sole and modified enzymes. The reduction of the $\Delta G_{\mathrm{H}_{2} \mathrm{O}}$ values for the modified enzyme in chemical denaturation indicated instability. A structural study by $C D$ and intrinsic fluorescence technique revealed the fluctuation of the secondary and tertiary structures of MT.
\end{abstract}

Keywords: modification, mushroom tyrosinase, Woodward reagent $K$, kinetics, structure.

Introduction. Mushroom tyrosinase (E.C. 1.14.18.1), as a polyphenol oxidase, is a multifunctional copper-containing enzyme from the oxidase superfamily. It is widely distributed in microorganisms, plants, and animals [1]. It plays a key role in melanin biosynthesis through the catalysis of two disparate reactions: the hydroxylation of monophenols (cresolase or monophenolase activity) and the oxidation of $o$-diphenols (catecholase or diphenolase activity) into reactive $o$-quinones [24]. Modification is a good method to recognize the role of residues in the structure and function of proteins. The polar amino acids, such as glutamate, aspartate, lysine, arginine, histidine, serine, tyrosine, methionine, and tryptophan, can be modified by chemical reagents. The affinity of amino acids for participating in the reactions depends on environmental conditions like $\mathrm{pH}$, the ionic power, the buffer type, and the nucleophilicity features [5]. The amino acid side-chains that participate in the enzymatic activity are usually accessible to the solvent and can be modified by the reagents that are especially accessible to proteins. Sometimes, according to the specific environment of the active site, we can design specific reagents. Chemical modification in the absence and presence of a ligand is a suitable method to recognize the side-chains of the amino acids' active sites [6]. In enzymes, the catalytic amino acid side-chains can be selectively modified by using the suicide substrates, and these can result in enzyme inactivity. The modification of amino acid side-chains plays an important role in an enzyme's catalytic activity [7]. MT has 576 residues, which can be divided into two parts. The residues numbering 1-392 form the chain part, and those numbering 393-576 form the propeptide part, which disappears in the mature form of MT. In the sequence of MT, the residues numbering 61, 85, and 94 are the binding sites of copper $\mathrm{I}\left(\mathrm{Cu}^{1+}\right)$, and those numbering 259 , 263, and 296 are the binding sites of copper II $\left(\mathrm{Cu}^{2+}\right)$. The mature MT includes 52 aspartic acid and glutamic acid residues in its sequence. The residues numbering 47, 50,269, 273, 289, and 300, which are aspartic acid, and the residues numbering 67, 98, 102, and 256, which are glutamic acid, are located near the histidine residues of the MT active sites [8,9]. The modification of these carboxyl groups by WRK causes enzymatic inactivation and the partial unfolding of the MT structure, leading to its instability.

Several studies have been done on the amino acid residues to reveal their respective roles in the function of enzymes. The modification of Tyr62 in lysozyme with N-bromosuccinimide caused a decrease in the catalytic velocity $\left(K_{\text {cat }}\right)$ of the enzyme [10]. In the other investigations, the role of different catalytic groups was considered by the modification of residues e.g., the conversion of $-\mathrm{SH}$ to $-\mathrm{OH}$ in cysteine residues in the active site of papain [11], the conversion of $-\mathrm{OH}$ to $-\mathrm{SH}$

*To whom correspondence should be addressed.

\footnotetext{
${ }^{a}$ Islamic Azad University Science and Research Branch, Department of Biology, Faculty of Basic Sciences, Tehran, Iran; ${ }^{b}$ Cellular and Molecular Research Center, Qazvin University of Medical Sciences, Qazvin, Iran; email: ngheibi@qums. ac.ir. Published in Zhurnal Prikladnoi Spektroskopii, Vol. 84, No. 6, pp. 969-976, November-December, 2017. Original article submitted June 20, 2016.
} 
in subtilisin $[12,13]$ and trypsin [14], and the conversion of $\mathrm{COOH}$ to $\mathrm{CONH}_{2}$ in lysozyme [15]. The modification of the histidine residue with diethylpyrocarbonate (DEP) in 2,3-dihydroxyphenylalanine decarboxylase revealed the importance of the histitidyl residue in the diethylpyrocarbonate activity in 2,3-D-hydroxyphenylalanine [16]. Also, the histitidyl residues in deoxynucleotide kinase bacteriophage T4 were altered with two modifiers, including DEP and pyridoxal-5"-phosphate. In addition, the reduction of enzymatic activity and the inhibition of enzyme even in high concentrations of modifiers have been reported [17]. The $\mathrm{Na}+$ channel in single myelinated nerve fibers was irreversibly inhibited after the modification of methionine residues by some reactive reagents, including N-bromoacetamide, N-bromosuccinimide, chloramine- , and N-chlorosuccinimide [18]. To continue the previous studies on the activity, stability, and structure of MT [19-21], we modified the MT by WRK and measured its activity, stability, and structural changes in this study to reveal the importance of the role of aspartic and glutamic acid residues in the enzyme.

Experiment. MT (EC 1.14.18.1) with a specific activity of 3400 units $/ \mathrm{mg}$ and WRK were bought from Sigma (Sigma-Aldrich). L-Dopa was bought from Merck, Germany. The buffer used in the assay and dialysis was $50 \mathrm{mM}$ PBS, $\mathrm{pH} 6.8$, with the salts obtained from Merck. All the experiments were carried out in $20^{\circ} \mathrm{C}$, and the solutions were prepared in doubly distilled water.

The carboxyl groups of MT were altered by WRK, a typical carboxyl group modifier, which was used to conduct the substrate kinetic reaction by using a 50-mM PBS (pH 6.8), incubated for an hour at $25^{\circ} \mathrm{C}$ and dialyzed by PBS in a sequential process for $48 \mathrm{~h}$. The [WRK]/[MT] ratios were $2.5,5,25$, and 50 .

The kinetic assays of catecholase were carried out by a Cary spectrophotometer, a 100 bio-model with jacketed cell holders. Freshly prepared enzyme and substrate solutions were used in this work. All enzymatic reactions were run in PBS $(50 \mathrm{mM})$ at $\mathrm{pH} 6.8$ in a conventional quartz cell thermostated to maintain the temperature at $20^{\circ} \mathrm{C}$. Selected conditions of solvent, buffer, $\mathrm{pH}$, temperature, and enzyme concentration were applied to assay the oxidase activity of MT according to the previous studies [22, 23]. In the catecholase reactions, the oxidation of L-Dopa was measured in $475 \mathrm{~nm}$ for 3 min using 40 units of MT in each reaction. The enzyme was modified using 2.5, 5, 25, and 50 ratios of [WRK]/[MT].

The stability of the enzyme under thermal stress conditions can be studied by internal fluorescence and an enzyme solution of $0.2 \mathrm{mg} / \mathrm{mL}$ for the native and modified MT. The excitation wavelength was $280 \mathrm{~nm}$, the maximum emission intensity wavelength was obtained at $340 \mathrm{~nm}$, and the thermal scan was performed in $10-90^{\circ} \mathrm{C}$. The chemical denaturation of the native and modified MT was carried out for enzyme titration using the titration of guanidine hydrochloride (GndHCL) as the denaturant. The thermal and chemical denaturation curves of the native and modified enzyme are depicted in Figs. 2 and 3. The magnitudes of the denaturation's Gibbs free energy $\Delta G^{0}$ as a criterion of the conformational stability of a globular protein are based on the two-Pace-state theory, as follows:

$$
\text { Native }(\mathrm{N}) \Leftrightarrow \text { Denatured (D) . }
$$

Considering the two-state mechanism for chemical and thermal denaturation of an enzyme and from the analysis of sigmoidal curves, the denatured fraction of enzyme $F_{\mathrm{d}}$ and the equilibrium constant $K$ can be determined by monitoring the changes in the absorbance or the fluorescent thermal intensity, as presented below:

$$
\begin{gathered}
F_{\mathrm{d}}=\left(Y_{\mathrm{N}}-Y_{\mathrm{obs}}\right) /\left(Y_{\mathrm{N}}-Y_{\mathrm{D}}\right), \\
K=F_{\mathrm{d}} /\left(1-F_{\mathrm{d}}\right)=\left(Y_{\mathrm{N}}-Y_{\mathrm{obs}}\right) /\left(Y_{\mathrm{obs}}-Y_{\mathrm{D}}\right),
\end{gathered}
$$

where $Y_{\mathrm{obs}}$ is the observed variable parameter (e.g., absorbance or thermal intensity) and $Y_{\mathrm{N}}$ and $Y_{\mathrm{D}}$ are the values of $Y$ characteristics of fully native and denatured conformation, respectively. The standard $\Delta G^{\mathrm{o}}$ change for the enzyme denaturation is given by the following equation:

$$
\Delta G=-R T \ln K
$$

where $R$ is the universal gas constant and $T$ is the absolute temperature. The quantity $\Delta G^{0}$ varies linearly with the denaturant concentration [GndHCL] and temperature $T$ over a limited region

$$
\Delta G^{\mathrm{o}}=\Delta G_{\mathrm{H}_{2} \mathrm{O}}^{\mathrm{o}}-m[\text { denaturant }] \text {, }
$$

where $\Delta G_{\mathrm{H}_{2} \mathrm{O}}^{\mathrm{O}}$ is the free energy of conformational stability in the absence of a denaturant, and $m$ is the value of $\Delta G^{\mathrm{o}}$ dependent on the denaturant concentration. The standard Gibbs free energy of enzyme denaturation at $25^{\circ} \mathrm{C}\left(\Delta G_{25^{\circ}} \mathrm{C}\right)$ can be obtained by the Pace analysis equation (5) [24]. The magnitudes of $\Delta G_{25^{\circ} \mathrm{C}}$ and $\Delta G_{\mathrm{H}_{2} \mathrm{O}}$ are the most important criteria of the enzyme conformational stability in the chemical and thermal denaturation process, respectively. 


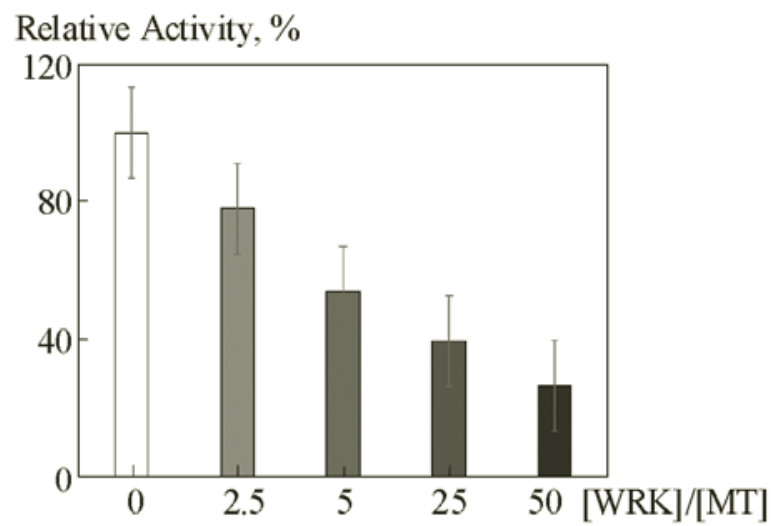

Fig. 1. Relative activity of native and $[\mathrm{WRK}] /[\mathrm{MT}]$ ratios $(2.5,5,25$, and 50$)$ through catecholase reaction and $1 \mathrm{mM}$ L-Dopa as substrate.

In chemical denaturation, $[\mathrm{GndHCL}]_{1 / 2}$ is the denaturant concentration needed for the enzyme to undergo half of its two-state transition. In thermal denaturation, the protein melting point $\left(T_{\mathrm{m}}\right)$ is the temperature needed for the enzyme

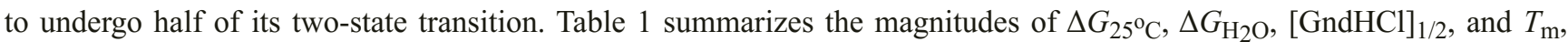
determined from the re-plots.

Intrinsic fluorescence analysis by the Stern-Volmer equation. The fluorescence intensities were recorded using a Cary Eclipse spectrofluorimeter at an excitation wavelength of $280 \mathrm{~nm}$, and the maximum emission wavelength was $340 \mathrm{~nm}$. A series of modified MT solutions was prepared with 2.5, 5, 25, and 50 ratios of [WRK]/[MT] in the 50-mM PBS. The equilibrium between the free and bound ligands (WRK) is given by the following equation to evaluate the type of binding interaction [25]:

$$
F_{0} / F=1+K_{\mathrm{SV}}[\mathrm{Q}]
$$

where $F_{0}$ and $F$ are the relative steady-state fluorescence intensities in the absence and presence of a quencher, $Q$ is the quencher (ligand) concentration, and the values of $K_{\mathrm{SV}}$ can be derived from the intercept and plot slope based on the above equation.

Circular dichroism measurement. The far-UV region (190-260 nm) corresponding to the secondary structure of MT was analyzed through an Aviv model 215 spectropolarimeter (Lakewood, USA). The far-UV spectra of MT were studied at the $0.2 \mathrm{mg} / \mathrm{mL}$ concentration with a 1-mm path-length quartz cell. Modified enzyme solutions were prepared in the 50-mM PBS at $\mathrm{pH}$ 6.8. The ellipticity of the enzyme solutions was obtained in the $[\mathrm{WRK}] /[\mathrm{MT}]$ ratios of $2.5,5,25$, and 50 . All spectra were collected in triplicate from 190 to $260 \mathrm{~nm}$, and the background was corrected against a buffer blank. The data were smoothed by the application of software, including the fast Fourier-transformed noise-reduction routine, which allows the enhancement of the most noisy spectra without distorting their peak shapes.

Results and Discussion. According to Fig. 1, the relative activity analysis through the catecholase reaction showed a decrease in the enzymatic activity from $100 \%$ for the native MT to $77.9,53.8,39.4$, and $26.4 \%$ for the MT modified by 2.5 , 5,25 , and 50 ratios of [WRK]/[MT], respectively, and $1 \mathrm{mM} \mathrm{L-Dopa} \mathrm{as} \mathrm{the} \mathrm{substrate.}$

The sigmoid curves (Fig. 2a) show the MT's thermal denaturation for the native enzyme and for the one modified with different [WRK]/[MT] ratios $(2.5,5,25$, and 50). The thermodynamic parameters related to the enzyme's stability — the melting point $\left(T_{\mathrm{m}}\right)$ and Gibbs free energy $\left(\Delta G_{25^{\circ} \mathrm{C}}\right)$ - were calculated according to the previous equations. The curves were derived from the thermal denaturation sigmoid curves for the native and modified forms of the enzyme (Fig. 2b). The $T_{\mathrm{m}}$ and $\Delta G_{25^{\circ} \mathrm{C}}$ values for the native and modified enzyme are shown in Table 1 . The chemical denaturation was performed in an optical density (OD) of $280 \mathrm{~nm}$ and $0.2 \mathrm{mg} / \mathrm{mL}$ of the enzyme solution, which was titered with GndHCL ( $8 \mathrm{M}$ stock solution) as a chemical denaturant at $20^{\circ} \mathrm{C}$. The chemical denaturation of the native and modified enzyme is depicted in Fig. 3a. The thermodynamic parameters - half of the denaturation concentration $\left(C_{\mathrm{m}}\right)$ and the Gibbs free energy $\left(\Delta G_{\mathrm{H} 2 \mathrm{O}}\right)$ - according to the previous relationships, and the curves (Fig. 3b) derived from the chemical denaturation sigmoid curves, have been calculated and are shown in Table 1.

The results of the fluorescence emission in Fig. 4a were referred to the tertiary structure of MT in its native and modified forms. Here, the set of curves shows that the intensities of the intrinsic fluorescence of MT decreased gradually with increase in the $[\mathrm{WRK}] /[\mathrm{MT}]$ ratio. The Stern-Volmer curve (Fig. 4b) is also drawn for the determination of the quenching 

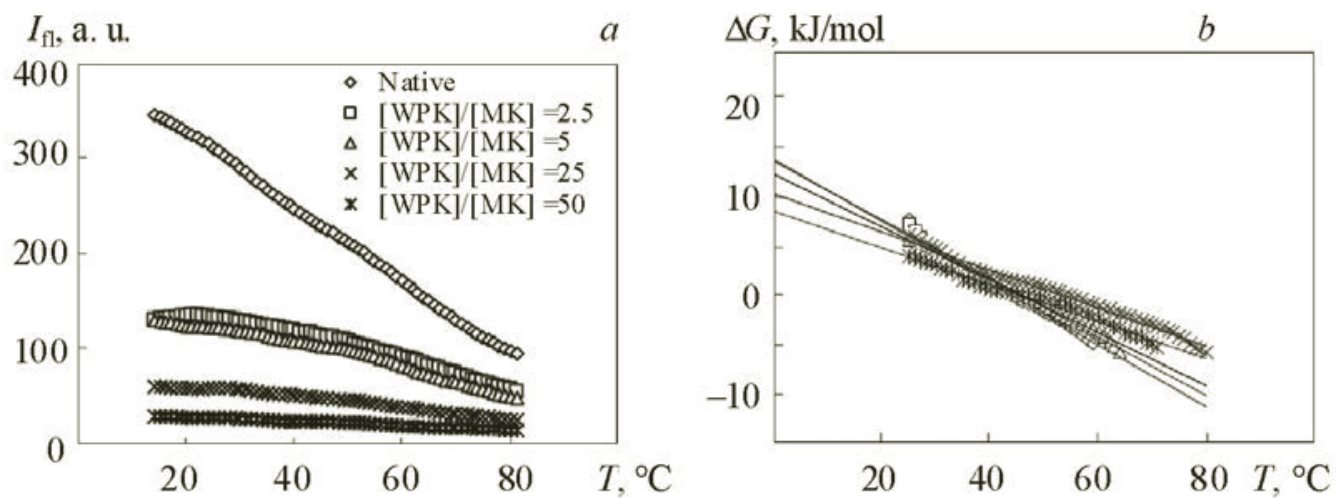

Fig. 2. Thermal denaturation profiles of MT obtained from intrinsic fluorescence intensity in $340 \mathrm{~nm}$ emission wavelength with temperature scanning (a) and linear secondary plots for obtaining the thermodynamic parameters $\Delta G_{25^{\circ} \mathrm{C}}$ and $T_{\mathrm{m}}(\mathrm{b})$ for native $(\diamond)$ and modified enzymes with $[\mathrm{WRK}] /[\mathrm{MT}]=2.5(\square), 5(\Delta), 25(\times)$, and $50(*)$
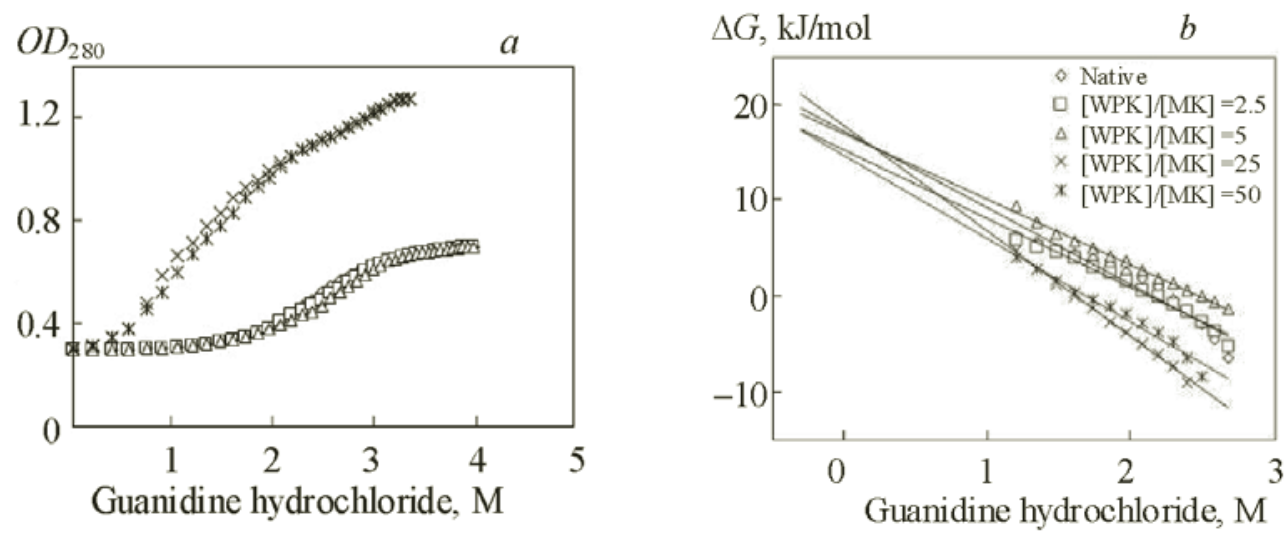

Fig. 3. Chemical denaturation profiles of MT obtained from $\mathrm{GnHCl}$ titration (a) and linear secondary plots for obtaining $\Delta G_{\mathrm{H}_{2} \mathrm{O}}$ and $C_{\mathrm{m}}$ as thermodynamic parameters (b) for native $(\diamond)$ and modified enzymes with $[\mathrm{WRK}] /[\mathrm{MT}]=2.5(\square), 5(\Delta), 25(\times)$, and $50(*)$.

type. The curve is dynamic. The CD study in the far-UV region (far-UV-CD) from 190 to $260 \mathrm{~nm}$, which is the peptidebond-absorbtion region, was done by a spectropolarimeter. For the study of the secondary structure of MT at a $0.2 \mathrm{mg} / \mathrm{mL}$ concentration, in the $50 \mathrm{mM}$ PBS buffer, and $\mathrm{pH} 6.8$, the spectra obtained with a repetition of three times and re-formed against the buffer as a blank by special software to decrease the noise via Fourier transform became smooth. In Fig. 5, the results are presented at elipticity $\theta$ (mdeg) for native and modified enzymes. According to Fig. 4, the internal fluorescence spectra of MT gradually decreased with increment of the $[\mathrm{WRK}] /[\mathrm{MT}]$ ratio, revealing the changes in the microenvironment of tryptophan and tyrosine residues and the quenching of these chromophores. By the excitation of MT in $280 \mathrm{~nm}$, the tryptophan and tyrosine residues were exposed and influenced. Then, the tertiary structure of the enzyme was transformed [26]. CD is exhibited in the absorption bands of optically active chiral molecules. The CD spectra of modified MT, compared to the native form in Fig. 5, show a minor change of regular secondary structures of modified MT. The overall modification of MT by WRK not only reduced the enzyme activity but also led to structural and thermodynamic instability. The previous investigations have reported the chemical modification of carboxyl groups with a carbodiimide reagent and isoxazolium salts (WRK) [27, 28]. Isoxazolium salts, especially N-ethyl-5-phenylisoxazolium-3"-sulfonate (WRK), can be used for the chemical modification of acidic amino acids, aspartate, and glutamate [29]. The reaction mechanism of WRK has been reported in [30]: its reaction with carboxyl groups produces enol ester. It is suggested that MT has two active sites. One of these is for substrate binding, and the other one, which is near the first site, is for the binding of the modifier or ligand [31]. The 
TABLE 1. Thermodynamic Parameters of Native and Modified MT

\begin{tabular}{|c|c|c|c|c|}
\hline \multirow{2}{*}{ WRK] $/[\mathrm{MT}]$} & \multicolumn{2}{|c|}{ Chemical denaturation } & \multicolumn{2}{c|}{ Thermal denaturation } \\
\cline { 2 - 5 } & {$[\mathrm{GndHCL}]_{1 / 2}, \mathrm{M}$} & $\Delta G_{\mathrm{H}_{2} \mathrm{O}}^{\mathrm{O}}, \mathrm{kJ} / \mathrm{mol}$ & $T_{\mathrm{m}},{ }^{\circ} \mathrm{C}$ & $\Delta G_{25^{\circ} \mathrm{C}, \mathrm{kJ} / \mathrm{mol}}$ \\
\hline Native & 8 & 17.22 & 61.2 & 19.37 \\
25 & 7.5 & 16.7 & 60.1 & 18 \\
5 & 6.6 & 15 & 58.3 & 15.7 \\
25 & 10.2 & 13.7 & 53.9 & 10.4 \\
50 & 8 & 11.5 & 42.5 & 8.5 \\
\hline
\end{tabular}

chemical modification of aspartate and glutamate residues in the MT structure via the reaction of WRK with their carboxyl groups involves the separation of a proton $\left(\mathrm{H}^{+}\right)$from the reagent with a base or water molecule and the formation of a very active and irreversible intermediate, ketoketenimine. From the structural aspect, this intermediate is a carbodiimide that reacts rapidly with carboxyl groups of MT and produces an enol ester that increases the maximum absorption at $340 \mathrm{~nm}$ [32-36]. The modification of Tyr62 in lysozyme by N-bromosuccinimide (NBS) not only causes an extreme reduction in binding ability, but also reduces the enzyme's catalytic velocity $\left(K_{\text {cat }}\right)[10]$. The thermodynamic denaturation of enzymes is the basic principle of MT stability. The denaturation of proteins is a process during which the native forms of the macromolecule change without the destruction of the primary covalent bonds. That is, in this process, the primary structure of the macromolecule (amino acid sequence) does not change but its conformation (3D building) does [37]. For the study of the tertiary structure, the effect of WRK on the intrinsic emission fluorescence of MT was studied. With increase in the modifier concentration, the fluorescence intensity decreased in all the samples. Because of the modifier interaction with the amino acids glutamate and aspartate, there was a change in the conformation. With changes in the conformation, the tryptophan residues were exposed and quenched by the WRK, so that the emission intensity decreased. This decrease in emission intensity demonstrated a change in the conformation and an instability of the modified enzyme. The quenching mechanism is usually classified as dynamic (collisional encounter between the fluorophore and quencher) or static (formation of a nonfluorescent ground state complex) [38], as shown in the Stern-Volmer curve (Fig. 4b). This curve is exponential; so, the type of interaction of the modifier with the binding site is dynamic. This means the modifier bound to the binding site was then separated, causing partial changes in the conformation of the modified enzyme and decreasing its activity, function, and stability.

As illustrated in Fig. 5, the values of the regular secondary structure ( $\alpha$-helix and $\beta$-sheet) were obtained by CD. With increase in the modifier concentration, the ellipticity values shifted toward the positive side, i.e., there was a decrease in the regular secondary structures. But here this decrease is not meaningful. So the WRK caused minor changes in the MT's secondary structures. A lot of investigation have been conducted into the different residues of proteins and enzymes modified by different chemical components. In this study, the aspartic and glutamic acid residues in MT were modified by WRK, and the results from the kinetic study showed a decrease in the activity and function of modified enzymes compared to the native form. Also, the internal fluorescence spectra in Fig. 4a show a change in the tertiary structure of the modified MT. This change in structure causes a decrease in the activity, function, and stability of the modified MT compared with the native form. The thermodynamic studies of the enzyme through chemical and thermal denaturation determined the magnitudes of $T_{\mathrm{m}}$ and $\Delta G$ for the native and modified enzymes. The results showed that the modified form of MT is unstable compared to the native MT. The results of this study show the importance of aspartic and glutamic acid residues in the activity, function, structure, and stability of MT. Like this study, several studies have reported that WRK reacts with the carboxylic groups of many proteins and enzymes with the appearance of a new absorbance peak at 330-350 $\mathrm{nm}$. Moreover, the modification of proton ATPase from rhodospirillum rubrum [39] and the inactivation of acylphosphatase by its modification with WRK have been reported [40]. The conformational changes of phosphoglycerate kinase were obtained by WRK concomitant with the enzyme's modification [41]. The modification of endoglucanase D from Clostridium thermocellum by WRK resulted in more than 95\% inactivation [42]. The modification of xylanase from alkalophilic Bacillus sp. by WRK showed almost $85 \%$ inactivation with the modification of two carboxylate groups. The substrate xylan protected the enzyme from inactivation, confirming that the carboxylate residues are in the active site. The $V_{\max }$ value of the modified xylanase decreased compared to the native form, while the $K_{\mathrm{m}}$ remained the same as the native, indicating the importance of carboxylate residues in the catalysis [43]. The investigation by Asthana et al. has shown the structure-activity relationships of two hydroxycoumarins 

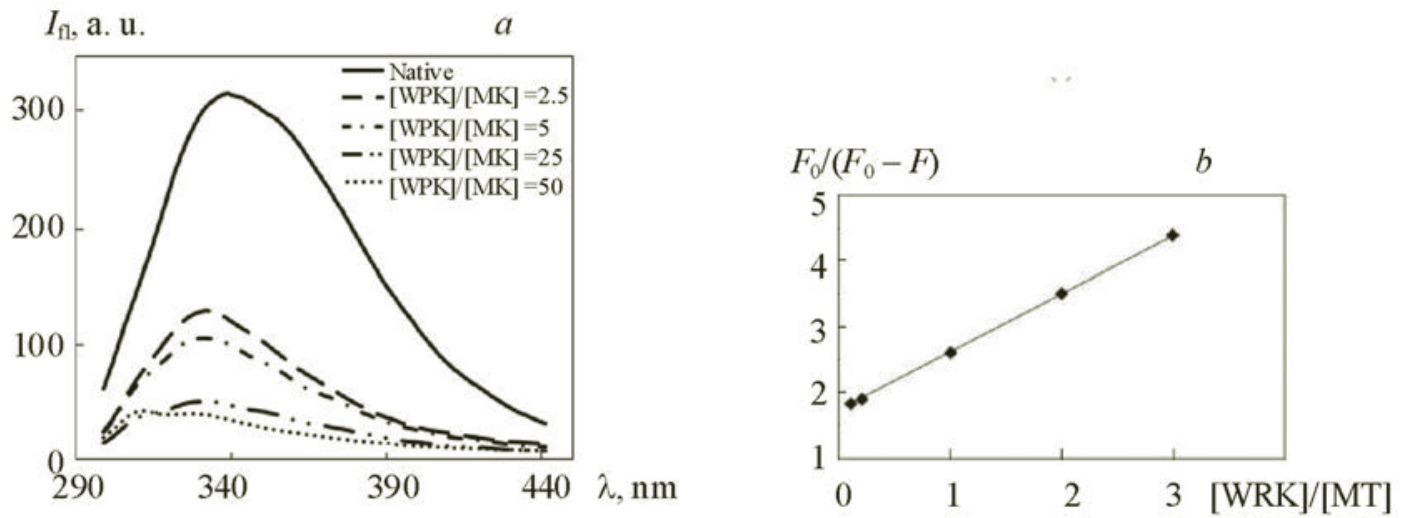

Fig. 4. Intrinsic fluorescence intensity of MT with the concentration of $0.2 \mathrm{mg} / \mathrm{mL}$ in the excitation wavelength of $280 \mathrm{~nm}$ and emission range of 300-450 $\mathrm{nm}$ for the native and modified enzymes (a); Stern-Volmer plot for quenching type determination (b).

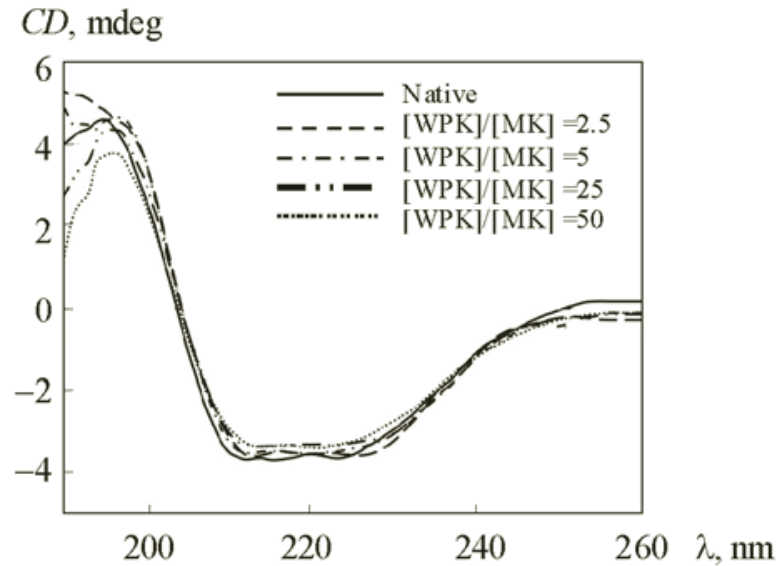

Fig. 5. Far-UV-CD spectra for native and modified enzymes.

with a hydroxy substituent on the aromatic ring of the molecule and their interactions with MT. In the maximum concentration, $88 \%$ inhibition has been reported [44]. In the study by Lin et al. [45], the tyrosinase was altered by two modifiers, DEP and bromoacetate, and the inactivation of tyrosinase by DEP and the reversible inactivation of enzyme by BrAce were reported.

Conclusions. The overall modification of MT by WRK not only reduced the enzyme's activity but also led to its structural and thermodynamic instability. The results of this study have shown the importance of aspartic and glutamic acid residues in the activity, function, structure, and stability of MT. Generally, the chemical modification of the carboxylic acid group of MT by WRK reduces the activity and stability of the modified enzyme compared with the native form and ascertains the role of the mentioned residues in MT's sequence. acknowledged.

Acknowledgment. The financial support of this work by Qazvin University of Medical Science is gratefully

The authors report no conflicts of interest. The authors alone are responsible for the content and writing of the paper.

\section{REFERENCES}

1. Y. Kim and H. Uyamab, Cell. Molec. Life Sci., 62, 1707-1723 (2005).

2. F. Canovas, F. Garcia-Carmona, V. Sanchez, J. Iborra Pastor, and J. Lozano-Teruel, J. Biol. Chem., 257, 8738-8744 (1982).

3. J. Rodriguez-Lopez, J. Tudela, R. Varon, and C. Garcia, Biochim. Biophys. Acta, 1076, 379-386 (1991). 
4. C. Cooksey, P. Garratt, and E. Land, J. Biol. Chem., 272, 26226-26235 (1997).

5. A. Glazer, R. Delange, and D. Sigman, North Holand/Elsevier, Amesterdam, 10-13 (1975).

6. P. Privalov, Crit. Rev. Biochem. Mol. Biol., 25, 281-305 (1990).

7. C. Walsh, Annu. Rev. Biochem., 53, 493-535 (1984).

8. W. Ismaya, H. Rozeboom, M. Schurink, C. Boeriu, H. Wichers, and B. Dijkstra, Acta Crystallogr., 67, 575-587 (2011).

9. W. Ismaya, R. Henriette, W. Amrah, M. Jurrian, F. Fabrizia, W. Harry, and D. Bauke, Biochemistry, 50, 5477-5486 (2011).

10. T. Imoto, M. Fujimoto, and K. Yagishita, J. Biochem., 76, 745-753 (1974).

11. P. Clark and G. Lowe, Eur. J. Biochem., 84, 293-299 (1978).

12. K. Neet and J. Koshland, Proc. Natl. Acad. Sci. USA, 56, 1606-1611 (1966).

13. L. Polgar and M. Bender, Biochemistry, 6, 610-620 (1967).

14. H. Yakosawa, S. Ojima, and S. Ishii, J. Biochem., 82, 869-876 (1977).

15. R. Kuroki, H. Yamada, T. Moriyama, and T. Imoto, J. Biol. Chem., 261, 13571-13574 (1986).

16. P. Dominici, B. Tancini, and V. Borri, J. Biol. Chem., 260, 10583-10589 (1985).

17. G. Brush and M. Bessman, J. Biol. Chem., 268, 1603-1609 (1993).

18. K. Ging, Biophys J., 46, 121-124 (1984).

19. N. Gheibi, A. Saboury, K. Haghbeen, F. Rajaei, and A. Pahlevan, J. Enzyme Inhibit. Med. Chem., 24, 1076-1081 (2009).

20. N. Gheibi, A. Saboury, K. Haghbeen, and A. Moosavi-Movahedi, J. Biosci., 31 (2006).

21. N. Gheibi, A. Saboury, H. Mansuri Torshizi, K. Haghbeen, and A. Moosavi-Movahedi, J. Enzyme Inhibit. Med. Chem., 20, 393-399 (2005).

22. N. Gheibi, A. Saboury, K. Haghbeen, and A. Moosavi-Movahedi, Colloids Surf B: Biointerfaces, 45, 104-107 (2005).

23. K. Haghbeen, M. Khalili, F. Nematpour, N. Gheibi, M. Fazli, M. Alijanianzadeh, S. Jahromi, and R. Sariri, J. Food Biochem., 34, 308-328 (2010).

24. C. Pace, B. Shiley, and J. Thomson, In Protein Structure: A Practical Approach, Ed. T. E. Creighton, Oxford, IRL, Vol. e9777, 311-330 (1990).

25. M. Eftink and C. Ghiron, Anal. Biochem., 114, 199-227 (1981).

26. N. Sun, S. Lee, and K. Song, Lebensmittel-Wissensch. Technol., 35, 315-318 (2002).

27. N. Carrilo, J. Arana, and R. Vallejos, J. Biol. Chem., 256, 6823-6828 (1981).

28. D. Dinur, E. Kantrowitz, and J. Hajdu, Biochem. Biophys. Res. Commun., 100, 785-792 (1981).

29. R. Woodward, R. Olofson, and H. Mayer, J. Am. Chem. Soc., 83, 1010-1012 (1961).

30. P. Petra, Biochemistry, 10, 3163-3170 (1971).

31. Q. Wang, Y. Shi, K. Song, H. Guo, L. Qiu, and Q. Chen, Protein J., 23, 303-308 (2004).

32. U. Sinha and J. Brewer, Anal. Biochem., 151, 327-333 (1985).

33. G. Meanst and R. Feeney, Bioconjug. Chem., 1, 2-12 (1990).

34. Y. Fujita, Y. Uraga, and E. Ichisima, Biochim. Biophys. Acta, 1261, 151-154 (1995).

35. C. Jimenes-Cervantes, J. Garcia-Barron, J. Lozano, and F. Solano, Biochim. Biophys. Acta, 1243, 421-430 (1995).

36. N. Chosa, T. Fukumitsu, K. Fujimoto, and E. Ohnishi, Insect Biochem. Mol. Biol., 27, 61-68 (1997).

37. S. Lapanje, Physiochemical Aspects of Protein Denaturation, John Wiley \& Sons, New York, 788-796 (1978).

38. J. Lakowicz, Principles of Fluorescence Spectroscopy, 3rd ed., Springer, New York, 278-285 (2006).

39. E. Ceccarelli and R. Vallejos, Arch. Biochem. Biophys., 224, 382-388 (1983).

40. P. Paolo, F.Tania, C. Paolo, C. Guido, M. Giampaolo, C. Gianni, R. Giovanni, M. Gloriano, and R. Giampietro, Biochem J., 328, 855-861 (1997).

41. J. Anthony and H. Bruce, J. Biol. Chem., 249, 5452-5457 (1974).

42. P. Tomme, J. Van Beeumen, and M. Claeyssens, Biochem J., 285, 319-324 (1992).

43. H. Balakrishnan, L. Satyanarayana, S. Gaikwad, and C. Suresh, Enzyme and Microbiol. Technol., 39, 67-73 (2006).

44. A. Shailendra, Z. Paolo, V. Attilio, S. Enrico, R. Paolo, and R. Antonio, J. Agric. Food Chem., 63, 7236-7244 (2015).

45. G. Lin, L. Zhi-Rong, P. Daeui, H. Sang, S. Long, J. Seong, B. Jong, P. Yong-Doo, R. Zhen-Long, and Z. Fei, J. Biomol. Struct. Dynam., 26, 395-401 (2008). 